\title{
Parásitos gastrointestinales en cachorros caninos provenientes de la venta comercial en el Cercado de Lima.
}

\author{
Gastrointestinal parasites in canine puppies for commercial sale in Lima downtown.

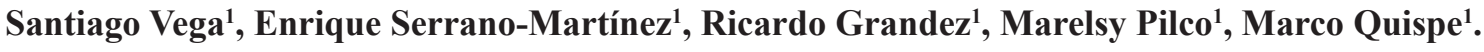

\section{RESUMEN}

Objetivo: Determinar la frecuencia de parásitos gastrointestinales en caninos menores de seis meses de edad provenientes de la venta comercial. También determinar si las variables raza, sexo y edad están asociadas a la presentación de cuadros de parasitosis. Metodología: Se recolectaron 97 muestras de heces de mascotas caninas que se comercializan en el cercado de Lima, las cuales fueron procesados mediante las técnicas de examen directo, de concentración por flotación, de concentración por sedimentación y de Ziehl Neelsen, considerándose positivas las muestras con presencia de huevos de parásito o parásitos bajo el microscopio. Resultados y Conclusiones: El $100 \%$ de las muestras analizadas fueron positivas a la presencia de parásitos. Las mayores frecuencias fueron del nemátode Toxocara canis fue $(87.96 \%)$ y del protozoario Isospora canis $(98.78 \%)$; los mismos como asociación biparasitaria (62.89\%) y monoparasitarias Toxocara canis (17.56\%); por primera vez se encontró una asociación de triprarasitismo de Toxocara canis - Isospora canis - Toxascaris leonina (12.37\%). En los machos hubo una mayor presentación de parasitosis $(57.7 \%)$ que en las hembras (42.3\%). Caninos de raza pura tuvieron mayor frecuencia de presentación de parasitosis (82.5\%); La edad no presentó diferencias estadísticas en los tres estratos estudiados, dándose en el estrato de $\geq 8 \mathrm{y}<12$ semanas representados por el $41.2 \%$ de casos. La parasitosis en caninos evaluados es del $100 \%$, siendo Toxocara canis el más frecuente.

PALABRAS CLAVE: caninos, parasitosis, parasito, huevos, frecuencia.

\begin{abstract}
Aim: To determine the frequency of gastrointestinal parasites in canines younger that 6 months for commercial sale in Lima downtown. Methods: 97 dog fecal samples were analyzed using the techniques of direct examination, concentration by flotation, by sedimentation and Ziehl Neelsen, it was considered a positive sample the presence of parasites or parasite eggs under the microscope. Results and Conclusions: $100 \%$ of the samples tested were positive for the presence of eggs or parasites, where the nematode Toxocara canis $(87.96 \%)$ and the protozoan Isospora canis $(98.78 \%)$ were found more frequently; and both of them as biparasitism association $(62.89 \%)$. Toxocara canis monoparasitism in (17.56\%). Toxocara canis - Isospora canis - Toxascaris leonina (12.37\%) triprarasitism association was found. In males there was a greater presence of parasites $(57.7 \%)$ than females $(42.3 \%)$. Pure breed had higher frequency of parasites (82.5\%); Age does not show statistical differences in the three strata studied. $41.2 \%$ of cases were in the range of $\geq 8$ and $<12$ weeks of age. The parasitism in the studied dogs is $100 \%$, being the most common Toxocara canis.
\end{abstract}

KEYWORDS: canines, parasites, egg, frequency.

Grupo SALUVET-UPCH, Facultad de Medicina Veterinaria y Zootecnia, Universidad Peruana Cayetano Heredia. Lima, Perú. 


\section{INTRODUCCIÓN}

La parasitosis es una de las patologías más frecuentes en humanos y animales, generando morbilidad y mortalidad, estos parásitos pueden permanecer por periodos largos en el organismo alterando la fisiología del hospedero (Acha, 2003. Boreham y Boreham, 1990). Muchas de estas son zoonóticas y dentro de este grupo podemos encontrar protozoarios, nemátodes y céstodes (Quiroz, 1999; Hendrix, 1999).

La Giardia sp. es un parásito zoonótico frecuente en niños y adultos causantes de gastroenteritis, la prevalencia en perros varía de un $10 \%$ al $100 \%$ afectando más a los cachorros que a los adultos (portadores asintomáticos) (Meloni et al., 1993. Cordero del Campillo y Rojo., 1999; Kirkpatrick, 1998). El Dipylidium caninum es un parásito del intestino delgado de perros, gatos, zorros y en ocasiones afecta al hombre, este céstodo tiene una distribución mundial y es frecuente en perros, los huéspedes intermediarios son las pulgas (Ctenocephalides canis, C. felis, Pulex irritans y el piojo Trichodectes canis) el huésped definitivo se parasita por ingesta de pulgas infectadas con metacéstodes, los proglótidos grávidos se eliminan con las heces; puede transmitir a los humanos y más susceptibles son los niños por la falta de higiene (Ferraris et al., 1993; Boreham y Boreham, 1990; Soulsby, 1987). En los perros, la presencia de $D$. caninum se caracteriza clínicamente por problemas digestivos, causa prurito en la región perianal y los signos clínicos varían dependiendo de la edad, el sexo, la raza y la condición física (Soulsby, 1987; Browman et al., 1995; Chappell et al., 1990). La toxocariasis es una zoonosis por la ingesta de huevos de Toxocara adultos que habitan en el intestino delgado de caninos y felinos; los parques contaminados con estos parásitos son un problema en salud pública (niños principalmente) al ingerir los huevos infectivos puede producir el Síndrome de la Larva Migrante Visceral (LMV) y Síndrome de la Larva Migrante Ocular (LMO) (Schanstz y Glickman, 1981; Leguia, 2002); también causan lesiones oculares (estrabismo, retinocoroiditis, uveítis) que conllevan a la pérdida de visión en forma progresiva o brusca (Acha, 2003).

En Buenos Aires Argentina se ha reportado seropositividad a $T$. canis en $67 \%$ de niños $(0$ a 16 años), con eosinofília mayor al $10 \%$, presentándose como (LMO) en un 6,7\% y (LMV) en 15,5\% de los casos y los pacientes seropositivos $(91,1 \%)$ tuvieron contacto con perros y/o gatos (López et al., 2005). El asma y la bronquitis recurrente se asocia a la presencia de anticuerpos contra Toxocara spp. También en La Patagonia, Bogotá, y Santa Catarina-Brasil, la presencia de parásitos zoonóticos se encuentran en espacios públicos como parques $(24,1$ a $90 \%)$ y plazas (28 a 90\%), siendo Toxocara spp. y Ancylostoma spp. los más frecuentes (Sánchez et al., 2003; Polo-Terán et al., 2007; Lettieri et al., 2003; López-Velez et al., 1996).

En el Perú, los estudios de contaminación ambiental en parques públicos han revelado que $T$. canis es más frecuente; estudios realizados en la Provincia Constitucional del Callao muestran una frecuencia de 37\% (Velarde, 1999), en el Cono Este 41\% (Serrano-Martínez, 2000), en el Cono Oeste 63\% (López, 1993), en el Cono Sur 29\% (Cajas, 1999) y en el Cono Norte 34\% (La Rosa et al., 2001) y San Martín de Porres tiene una frecuencia de $T$. canis de 33,3\% (Chávez, 2002). Los niños son más susceptibles a parasitosis intestinal debido a la falta de higiene, sobre todo en la etapa escolar, la deficiencia de hierro y anemia también se reportan como una de las manifestaciones clínicas producidas por parásitos, debilitando el sistema inmune, la capacidad física y mental; en personas adultas, especialmente en mujeres embarazadas, se ha reportado anemia, debilidad y letargia por Trichuris sp. (82,25\%), Anquilostoma sp. $(47,22 \%)$ y Áscaris sp. $(63,92 \%)$ (Larocque et al., 2005; Marcos et al., 2003; Amaya et al., 2006; Villota, 1997; Vásquez et al., 2002).

Debido a que los niños constituyen la población con mayor riesgo de exposición a parásitos de potencial zoonótico. Por la estrecha relación entre niño y mascota, es el grupo poblacional que con frecuencia puede encontrarse infectado; además de tener contacto directo con ambientes contaminados con huevos y ooquistes infectivos en parques o áreas de esparcimiento. El objetivo del estudio fue determinar la frecuencia de parásitos gastrointestinales en mascotas caninas menores de seis meses provenientes de la venta comercial en el cercado de Lima.

\section{MATERIALES Y MÉTODOS}

\section{Lugar de estudio}

El estudio se desarrolló en los establecimientos que están ubicados en el Cercado de Lima, entre la avenida Abancay, el jirón Ucayali, jirón Andahuaylas y jirón Puno, comprendiendo seis manzanas, donde se realiza 
tradicionalmente la comercialización de mascotas caninas y otras especies.

Las muestras de heces de cachorros fueron analizadas en el Laboratorio de Parasitología en la Facultad de Veterinaria y Zootecnia de la Universidad Peruana Cayetano Heredia.

\section{Diseño del estudio y tamaño de muestra}

El tamaño de muestra se determinó mediante el programa WINEPISCOPE 2.0 utilizando la fórmula de comprobación de una proporción de una población, utilizando las restricciones de prevalencia previa desconocida de $50 \%$ para infecciones parasitarias en caninos (valor usado al desconocer un valor de prevalencia referencial) con un nivel de confianza del $95 \%$ y un error máximo admisible del $10 \%$.

El tamaño de muestra tomado para el estudio de frecuencia de parásitos gastrointestinales fue de 97 cachorros.

\section{Recolección de muestras e identificación de los mismos}

Las muestras fecales se recogieron del suelo del canil, la edad de los canes (menores de seis meses) se calculó a partir de los datos proporcionados por los vendedores, tiempo de estadía en el establecimiento y dentición de los animales. Todas las muestras se rotularon y se almacenaron en cajas de teckno-port, hasta ser llevados al laboratorio manteniendo la cadena de frío de $-4{ }^{\circ} \mathrm{C}$.

\section{Procesamiento de muestras:}

Las muestras se analizaron mediante las técnicas del examen directo, de concentración por flotación, de concentración por sedimentación y de Ziehl Neelsen.

\section{Técnica de examen directo:}

En un portaobjetos se colocó por separado una gota de solución salina fisiológica $(0.85 \%)$ y otra de lugol. Se tomó con un palillo una pequeña porción de materia fecal y se hizo una suspensión en la gota de solución salina igualmente se realizó en el lugol. Se cubrió con una lámina cubre objeto y se observó al microscopio con objetivo de 100X y 400X.

\section{Técnica de concentración por flotación:}

En un mortero se depositó una cantidad máxima de 3 gramos de heces, se mezcló con un poco de agua corriente y de estos $2 \mathrm{ml}$, usando una pipeta de Pasteur, se añadió a un tubo de ensayo. Al tubo de ensayo se agregó una cantidad de solución saturada de sal hasta llegar al tope formando una superficie convexa.

Luego se colocó una lámina cubre objetos y se dejó reposar unos 15 a 20 minutos (los huevos de parásitos flotan hacia la lámina cubreobjetos) y se observó al microscopio a 100X y 400X.

\section{Técnica de concentración por sedimentación}

En un mortero se depositó 3 gramos de heces y se mezcló con agua corriente. Luego, la mezcla se depositó en un embudo tamizado que se colocó dentro de una copa. Posteriormente, se agregó agua corriente hasta $3 \mathrm{ml}$ antes de llenar la copa. Se dejó reposar 15 minutos y se eliminó el sobrenadante, enseguida se agregó agua corriente y se dejó reposar 15 minutos otra vez. Este procedimiento se repitió dos veces más. Finalmente quedó el sobrenadante, del cual se obtuvo una gota con una pipeta Pasteur, y se colocó en una lámina portaobjeto. Ésta se cubrió con una lámina cubre objeto y se observó al microscopio a $100 \mathrm{X}$ a 400X.

\section{Técnica de tinción de Ziehl Neelsen}

La muestra se frotó con un hisopo sobre el portaobjetos, se añadió Fucsina Básica cubriendo todo el portaobjetos por unos 5'. Se eliminó el exceso de Fucsina con agua corriente a baja presión, evitando que la película adherida de la muestra se desprenda, luego se añadió Azul de Metileno por todo el porta objeto por 5'; se volvió a eliminar el exceso del colorante con agua a baja presión, se dejó secar la lámina para y se observó al microscopio con objetivo de $100 \mathrm{X}$ a 400X.

\section{Desarrollo de la base de datos}

La información obtenida de los cachorros (de la edad, sexo y raza) sumado al diagnóstico coproparasitológico, fue transferida a una base de datos en hojas de cálculo del programa Microsoft Excel 2010. El lenguaje utilizado en ambas informaciones fue numérico con la finalidad de facilitar los cálculos estadísticos y la leyenda correspondiente se almacenó en una hoja aparte.

\section{Análisis de datos}

Los resultados de frecuencias fueron expresados en forma porcentual considerando las variables (sexo, raza puras y mestizos y edad, clasificado en 3 estratos: $<8$ semanas, $\geq 8$ semanas $\mathrm{y}<12$ semanas, $\geq 12$ semanas). También se analizó por Chi Cuadrado para 
observar su posible asociación con la parasitosis y la relación de resultados entre técnicas fue analizada con la Prueba de Kappa y Mc Nemar usando el programa estadístico SPSS 15.0.

\section{RESULTADOS Y DISCUSIÓN}

Se analizaron 97 muestras fecales, el $100 \%$ resultaron positivas a presencia de huevos de parásito o parásitos, con mayor frecuencia se encontró a Toxocara canis $(87.96 \%)$ y al protozoario Isospora canis $(98.78 \%$ ) (Tabla 1), estos resultados son elevados en comparación con el estudio de Plascencia (2011), esto puede atribuirse a que no existe un plan de desparasitación debido a que son manejados por comerciantes que desconocen el programa sanitario, desparasitan a todo los cachorros con el mismo fármaco para economizar y muchas veces aplican sub dosis o confunden una parasitosis con una gastroenteritis, desencadenando la parasitosis crónicas y reinfección. Además la frecuencia de Toxócara canis puede deberse a que existe una mayor probabilidad de transmisión vertical de parásitos (Leguía, 2002). Los cachorros siguen ingiriendo larvas de Toxocara a través de la leche materna o cuando la madre lo lame, en el interior de estos los huevos de Toxocara maduran a la fase larvaria, atraviesan la pared intestinal, migrando al hígado hasta los pulmones (confundiéndose con cuadros virales) para madurar (Lloria, 2001). Es reconocido que la prevalencia de parasitosis en cachorros varía del 10\% al 100\%, debido a que las hormonas propias de la gestación originan la inmuno relajación peri-parto que estimulan a las larvas (inactivas o arrestada en los tejidos) migrar hacia el útero, glándulas mamarias o hacia los fetos en desarrollo (Lloria, 2001); es importante remarcar que los animales del estudio de Plasencia (Plascencia, 2011) provenían de propietarios, donde posiblemente se realizó la desparasitación como parte de su programa de salud; a diferencia de los animales de este estudio que carecen de manejo sanitario.

La Isospora sp. Constituyó el segundo parásito en importancia (98.78\%) siendo este parásito de carácter zoonótico (López et al., 2006), a la vez estos parásitos son resistentes a diferentes condiciones medioambientales y abundan en donde existen caninos (Leguía, 2002; Quiroz, 1999). Este parásito tiene la capacidad de invadir las células de la mucosa del intestino delgado de todo vertebrado en el perro es considerado hospedero definitivo, mientras que los gatos al ingerir los ooquistes esporulados, los esporozoítos se enquistan en los tejidos (Tananta, 2002)

Dentro de las asociaciones, las infecciones biparasitarias (65.98\%) de Toxocara canis e Isospóra canis fueron los más frecuente $(62.89 \%)$; en las monoparasitarias $(18.56 \%)$ se evidenció a Toxocara canis $(17.53 \%)$ y en el triparasitismo se encontró a Toxocara canis - Isospóra canis - Toxascaris leonina $(12.37 \%)$ con 12 muestras positivas (Tabla 2) estos cuadros de parasitismo similares a los resultados obtenidos en el estudio de Plasencia (Plascencia, 2011), (78.8\%). Además por primera vez se encontró dos presentaciones de triparasitismo como Toxocara canis - Isospóra canis - Dipylidium caninum y Toxocara canis - Isospóra canis - Toxascarias leonina, este tipo

Tabla 1: Porcentaje de parásitos identificados en 97 caninos menores de seis meses provenientes de la venta comercial como mascotas en el Cercado de Lima.

\begin{tabular}{lcc}
\hline PARÁSITOS & $\begin{array}{c}\text { Muestras Positivas } \\
(+)\end{array}$ & $\begin{array}{c}\text { Porcentaje } \\
(\mathbf{\%})\end{array}$ \\
\hline NEMÁTODOS & & \\
Toxocara canis & 95 & 87.96 \\
Toxascaris leonina & 13 & 12.04 \\
TOTAL & $\mathbf{1 0 8}$ & $\mathbf{1 0 0}$ \\
CÉSTODOS & & \\
Dipylidium caninum & 4 & 100 \\
TOTAL & $\mathbf{4}$ & $\mathbf{1 0 0}$ \\
PROTOZOARIOS & & \\
Isospora canis & 81 & 98.78 \\
Cryptosporidium parvum & 1 & 1.22 \\
TOTAL & $\mathbf{8 2}$ & $\mathbf{1 0 0}$ \\
\hline
\end{tabular}


Tabla 2: Distribución de asociaciones parasitarias en 97 caninos menores de seis meses provenientes de la venta comercial como mascotas en el Cercado de Lima.

\begin{tabular}{|c|c|c|}
\hline PARÁSITO. & $(+)$ & $(\%)$ \\
\hline \multicolumn{3}{|l|}{ MONOPARASITISMO } \\
\hline Toxocara canis & 17 & 17.53 \\
\hline Isospora canis & 1 & 1.03 \\
\hline TOTAL & 18 & 18.56 \\
\hline \multicolumn{3}{|l|}{ BIPARASITISMO } \\
\hline Toxocara canis - Isospora canis & 61 & 62.89 \\
\hline Toxocara canis - Dipylidium caninum & 1 & 1.03 \\
\hline Toxocara canis - Toxascaris leonina & 1 & 1.03 \\
\hline Toxocara canis - Cryptosporidium & 1 & 1.03 \\
\hline TOTAL & 64 & 65.98 \\
\hline \multicolumn{3}{|l|}{ TRIPARASITISMO } \\
\hline Toxocara canis - Isospora canis - Dipylidium caninum & 3 & $3.09^{\mathrm{a}}$ \\
\hline Toxocara canis - Isospora canis - Toxascaris leonina & 12 & 12.37 \\
\hline TOTAL & 15 & 15.46 \\
\hline
\end{tabular}

Tabla 3: Porcentajes de muestras positivas y su asociación según variables sexo, raza y edad en 97 caninos menores de seis meses provenientes de la venta comercial como mascotas en el Cercado de Lima.

\begin{tabular}{lcc}
\hline Variables & (n) & (\%) \\
\hline Sexo & & \\
Macho & 56 & 57.7 \\
Hembra & 41 & 42.3 \\
Raza & & \\
Puro & 80 & $82.5 \mathrm{a}$ \\
Mestizo & 17 & $17.5 \mathrm{~b}$ \\
Edad (rango/semanas) & & \\
$<8$ & 26 & 26.8 \\
$\geq 8$ y $<12$ & 40 & 41.2 \\
$\geq 12$ y $<16$ & 31 & 32 \\
total & $\mathbf{9 7}$ & $\mathbf{1 0 0}$ \\
\hline
\end{tabular}

a y b: Diferencias significativas

de presentación confirma que los cachorros menores de seis meses en condiciones sanitarias deficientes son susceptibles de desencadenar cuadro de parasitosis de moderado a severo, dado que los caninos positivos a triparasitismo durante la inspección mostraron pobres condiciones físicas comparados a los que presentaron monoparasitismo y biparasitismo.

En lo que respecta al sexo, se observó una frecuencia de parasitismo de $57.7 \%$ en machos (56 individuos) y $42.3 \%$ en hembras (41 individuos), se observó que la parasitosis tuvo una mayor presentación en machos que en hembras.

Con respecto a la raza, los cachorros puros presentaron $82.5 \%$ de parasitismo y $17.5 \%$ en mestizos, esto puede atribuirse a que los de raza pura son más sensibles debido a las manipulaciones genéticas, para la selección de características fenotípicas disminuyendo características genotípicas, disminuyendo las 


\begin{tabular}{|c|c|c|c|c|c|c|c|c|}
\hline $\begin{array}{l}\text { Variable } \\
\text { Edad }\end{array}$ & $\begin{array}{c}\text { Toxocara } \\
\text { (n) }\end{array}$ & $\begin{array}{l}\text { canis } \\
(\%)\end{array}$ & $\begin{array}{c}\text { Isospora } \\
\text { (n) }\end{array}$ & $\begin{array}{c}\text { Canis } \\
(\%)\end{array}$ & $\begin{array}{c}\text { Toxascaris } \\
\text { (n) }\end{array}$ & $\begin{array}{c}\text { leonina } \\
(\%)\end{array}$ & $\begin{array}{l}\text { Dipylidium caninum } \\
\text { (n) }\end{array}$ & $\begin{array}{c}\text { Cryptosporidium parvum } \\
(\%)\end{array}$ \\
\hline \multirow{2}{*}{$<8$ sem } & 25 & 25.77 & 24 & 24.74 & 3 & 3.10 & 0 & 0.00 \\
\hline & 1 & 1.03 & 2 & 2.06 & 23 & 23.71 & 26 & 26.80 \\
\hline \multirow{2}{*}{$\geq 8 \mathrm{Sem}$} & 40 & 41.24 & 33 & 34.02 & 15 & 15.46 & 1 & 1.03 \\
\hline & 0 & 0.00 & 7 & 7.22 & 25 & 25.77 & 39 & 40.20 \\
\hline \multirow{2}{*}{$>12-<16 \mathrm{Sem}$} & 30 & 30.93 & 24 & 24.74 & 4 & 4.12 & 3.10 & 0.00 \\
\hline & 1 & 1.03 & 7 & 7.22 & 27 & 27.84 & 28.87 & 31.96 \\
\hline
\end{tabular}

capacidades fisiológicas, susceptibles al estrés y/o cambios de hábitats.

En relación al grupo etario no hubo diferencias estadísticas significativas, observándose mayor parasitosis en el estrato $\geq 8 \mathrm{y}<12$ semanas $(41.2 \%)$ (Tabla 3), posiblemente se debe a la existencia de mayor número de individuos, considerando que es la edad cercana al post-destete, donde ya comen solos y es la edad ideal para la comercialización de cachorros.

\section{Correspondencia:}

Enrique Serrano-Martínez

enrique.serrano@upch.pe

\section{REFERENCIAS BIBLIOGRÁFICAS}

1. Acha P, Szyfres B. 2003. Parasitosis en: Zoonosis $\mathrm{y}$ enfermedades transmisibles comunes al hombre y a los animales. Vol. 3. $3^{\text {ra }}$ Ed. Washington. Organización Panamericana de la Salud. p351.

2. Boreham R, Boreham P. 1990. Dipylidium caninum: Life cycle, epizootiology and control. Compendium on Continuing Education for the Practicing Veterinarian, 12(5): 667-675.

3. Cajas J. 1999. Estudio de la contaminación de parques públicos con huevos de Toxocara canis en los distritos del Cono Sur (Chorrillos, San Juan de Miraflores, Villa María del Triunfo y Villa el Salvador). Tesis de Bachiller en Medicina Veterinaria. Lima. Universidad Nacional de San Marcos. p 77.

4. Chappell C, Enos J, Penn H. 1990. Dipylidium caninum, an under-recognized infection in infants and children. Pediatric Infectious Disease Journal, 9(10), 745-747.

5. Cordero del Campillo M, Rojo Vázquez F. 1999. Parasitología Veterinaria. McGraw Hill. México. p 77-78; 221-222; 620-623.

6. Ferraris S, Reverso E, Parravicini L. 1993. Dipylidium caninum in an infant. European journal of pediatrics 152(8): 702-702.

7. Hendrix Ch. 1999. Diagnostico parasitológico veterinario. 2a ed. Madrid: Harcourt Brack. p326.
8. Kirkpatrick C. 1988. Veterinary parasitology 30(2): 113-124.

9. La Rosa V, Chávez A, Casas E. 2001. Contaminación de parques públicos con huevos de Toxocara spp. Tesis de Bachiller en Medicina Veterinaria. Lima. Universidad Nacional Mayor de San Marcos. 12p

10. Larocque R, Casapia M, Gotuzzo E, Gyorkos T. 2005. Relationship between intensity of soiltransmitted helminth infections and anemia during pregnancy. The American journal of tropical medicine and hygiene, 73(4): 783-789.

11. Leguía G. 2002. Enfermedades parasitarias de perros y gatos. 2a ed. Lima: Ed. Del Mar. p155.

12. Lettieri M, Rossi L, De Freitas L, Gasparin N, Piva S, Meneghello A. 2008. Prevalence of Toxocara canis infection in public squares of the Concórdia City, Santa Catarina, Brazil. Parasitología latinoamericana, 63: 69-71.

13. Lopez T. 2003. Estudio de la contaminación de parques públicos con huevos de Toxocara canis en los distritos del Cono Oeste. Tesis de Bachiller en Medicina Veterinaria. Lima. Universidad Nacional de San Marcos. p87.

14. López-Velez R, Turrientes M, Malo Q, Feno M. 1996. 2 cases of toxocariasis. Enfermedades Infecciosas y Microbiología Clínica 14:.548-550.

15. López A, Martin G, Chamorro M, Alonso J. 2005. Toxocariosis en niños de una Región Subtropical. Medicina (Buenos Aires) 65: 226-230.

16. López J, Abarca K, Paredes P, Inzunza E. 2006. Parásitos intestinales en caninos y felinos con cuadros digestivos en Santiago, Chile. Consideraciones en Salud Pública. Revista médica de Chile 134: 193200.

17. Lloria M. 2001. Endoparasitos en animales de compañía. Prevención, Farmacia Profesional. p109.

18. Marcos L, Maco V, Terashima A, Samalvides F, Miranda E, Gotuzzo E. 2003. Parasitosis intestinal en poblaciones urbana y rural en Sandia, Departamento de Puno, Perú. Parasitología latinoamericana 58: 3540.

19. Meloni B, Thompson R, Hopkins R, Reynoldson J, Gracey M. 1993. The prevalence of Giardia and other intestinal parasites in children, dogs and cats from aboriginal communities in the Kimberley. The 
Medical Journal of Australia, 158(3), 157-159.

20. Polo-Terán L, Cortés-Vecino J, Villamil-Jiménez L, Prieto E. 2007. Contaminación de los Parques Públicos de la Localidad de Suba, Bogotá con Nemátodos Zoonóticos Revista de salud pública. 9 (4):550-557.

21. Plascencia L. 2011. Frecuencia de parásitos gastrointestinales en mascotas caninas (Canis familiaris) y evaluación de prácticas asociadas a su exposición en escolares de nivel primario del distrito de San Martín de Porres. Tesis Médico Veterinario y Zootecnista. Universidad Peruana Cayetano Heredia.

22. Quiroz H. 1999. Parasitología. 4a ed. México: Limusa. p876.

23. Sánchez P, Raso S, Torrecillas C, Mellado I, Nancufil N, Oyarzo C, Basualdo J. 2003. Contaminación biológica con heces caninas $\mathrm{y}$ parásitos intestinales en espacios públicos urbanos en dos ciudades de la Provincia del Chubut: Patagonia Argentina. Parasitología latinoamericana, 58(3-4), 131-135.

24. Serrano-Martínez E. 2000. Estudio de la contaminación de parques públicos con huevos de Toxocara spp. en los distritos del Cono Este (Ate Vitarte, Chaclacayo, Cieneguilla, El Agustino, La Molina, San Juan de Lurigancho, Santa Anita). Tesis de Bachiller en Medicina Veterinaria. Lima: Universidad Nacional Mayor de San Marcos. p73.

25. Schanstz P, Glickman L. 1981. Epidemiology and pathogenesis of zoonotic toxocariasis. Epidemiologic Reviews 3(1): 230-250.
26. Soulsby E. 1987. Parasitología y enfermedades parasitarias en los animales domésticos. 7a ed. México: Interamericana. p 102-123.

27. Tananta V. 2002 Presencia de enteroparasitos en lechuga (lactuca sativa) en establecimiento de consumo público de alimentos del Cercado de Lima. Tesis de Bachiller en Medicina Veterinaria. Universidad Nacional Mayor de San Marcos. p58.

28. Vásquez-Garibay E, Romero-Velarde E, NápolesRodríguez F, Nuño-Cosío M, Trujillo-Contreras F, Sánchez-Mercado O. 2002. Prevalencia de deficiencia de hierro y yodo, y parasitosis en niños de Arandas, Jalisco, México. Salud Pública México 44 (3): 195200.

29. Velarde J. 1999. Contaminación de los parques públicos de la provincia constitucional del Callao con huevos de Toxocara spp. Tesis de Bachiller en Medicina Veterinaria. Universidad Nacional Mayor de San Marcos. p62.

30. Villota M. 1997. Relación de los factores socioeconómicos, conocimientos y costumbres de las madres de menores de 5 años con parasitosis intestinal. AA.HH. Perpetuo Socorro - Rímac. Tesis de Licenciatura en Enfermería. Universidad Peruana Cayetano Heredia. p63.

Recibido: 17/06/2014

Aceptado: 22/12/2014 